\title{
Clinical efficacy of acetylcysteine combined with tetrandrine tablets on patients with silicosis and its effect on exercise tolerance and pulmonary function
}

\author{
XIUZHI GUO ${ }^{1}$, JING $\mathrm{QI}^{1}$, HUANXIANG $\mathrm{LI}^{2}$ and ZHAOFENG XING ${ }^{3}$ \\ ${ }^{1}$ Department II of Occupational Disease; ${ }^{2}$ Department of Public Health; ${ }^{3}$ Occupational Disease Diagnosis, \\ Branch of Tai'an City Central Hospital, Tai'an, Shandong 271000, P.R. China
}

Received July 31, 2018; Accepted December 3, 2019

DOI: $10.3892 /$ etm.2020.8858

\begin{abstract}
Clinical efficacy of acetylcysteine combined with tetrandrine tablets in the treatment of silicosis was investigated to observe the exercise tolerance and pulmonary function of the patients. The clinical data of 248 cases of silicosis patients treated in Branch of Tai'an City Central Hospital were retrospectively analyzed in this study, including 116 cases with conventional therapy (control group), and 132 cases treated with acetylcysteine and tetrandrine tablets on the basis of conventional therapy (observation group). The chest tightness, chest pain, cough and sputum production were evaluated by the St. George Respiratory questionnaire (SGRQ); and side effects after treatment were recorded. Exercise tolerance was measured by 6 -min walk test (6MWT) and pulmonary function was measured by pulmonary function instrument. The symptoms of chest tightness, chest pain, cough and sputum production in the observation group and the control group after 8 months of treatment were significantly lower than those before treatment $(\mathrm{P}<0.05)$, and that in the observation group after treatment was significantly higher than that in the control group $(\mathrm{P}<0.05)$. The incidence of side effects of the drug in the observation group was significantly lower than that in the control group $(\mathrm{P}<0.05)$. The $6 \mathrm{MWT}$, forced vital capacity (FVC) and forced expiratory volume in $1 \mathrm{sec}$ (FEV1) in the observation group and the control group after treatment were significantly higher than those before treatment $(\mathrm{P}<0.001)$; the 6MWT in the observation group after treatment was significantly higher than that in the control group $(\mathrm{P}<0.001)$. In conclusion, acetylcysteine combined with tetrandrine tablets on the basis of conventional therapy in treatment of silicosis is more effective than conventional therapy alone,
\end{abstract}

Correspondence to: Dr Zhaofeng Xing, Occupational Disease Diagnosis, Branch of Tai'an City Central Hospital, 336 Wanguan Avenue, Tai'an, Shandong 271000, P.R. China

E-mail: xau3pe@163.com

Key words: acetylcysteine, tetrandrine tablets, silicosis, exercise tolerance, pulmonary function which can also improve exercise tolerance, pulmonary function and clinical symptoms such as chest tightness, chest pain, cough, sputum production of silicosis patients, and is helpful for clinical application.

\section{Introduction}

Most silicosis patients are quarrying, coal mine, glass, ceramics factory workers because silicosis is an occupational disease caused by long-term exposure and inhalation of silica dust $(1,2)$. Silica dust enters the respiratory tract and alveoli by breathing, causing inflammation in the lungs, and then causing pulmonary fibrosis, which seriously affects the pulmonary function (3). According to statistics, the number of silicosis patients in developing countries has exceeded 600,000; the number of new cases has increased rapidly, and the rate of death from silicosis continues to increase (4). Silicosis is an irreversible chronic disease that continues to deteriorate. The treatment is based on the principle of avoiding complications as far as possible and needs long-term medication, and the quality of life of patients will be seriously affected with the development of the disease (5). The silicotic lung has the characteristics of high medical costs, high disability rate and high mortality rate, heavy financial burden and psychological pressure on the patient's family, and no special drugs have been developed for the treatment of silicosis so far, therefore, the treatment can only be the basis of controlling and delaying the development of the disease, and the main purpose is to improve the immune function and quality of life of patients at the same time (6). Dyspnea in silicosis patients is caused by obstruction of the airway, while the relevant Chinese literature shows that 6-min walk test (6MWT) is related to dyspnea, which can be used as an index to evaluate patient's exercise tolerance. 6MWT is currently an objective evaluation index for exercise tolerance in patients with chronic obstructive pulmonary disease, respiratory failure and other lung diseases $(7,8)$.

Acetylcysteine, the main active component of acetylcysteine effervescent tablets, is widely used in the treatment of many respiratory diseases, such as bronchial asthma, chronic obstructive pulmonary disease because it can dissolve concentrated phlegm (9). In addition, it has been reported that acetylcysteine has antioxidant and inhibitory effects 
on neutrophil chemotaxis in bronchial mucosa of silicosis patients (10). Tetrandrine, as an alkaloid, plays a major role in analgesia, anti-inflammation, and can effectively improve pulmonary vascular smooth muscle spasm, thereby improving pulmonary microcirculation, thus reducing clinical features such as cough and chest tightness in patients $(11,12)$.

Because most of the previous studies focused on lung ventilation and pulmonary function in silicosis patients, the clinical efficacy of acetylcysteine combined with tetrandrine tablets on the basis of conventional therapy in treatment of silicosis and the observation of exercise tolerance and pulmonary function in patients were conducted in this study to provide a certain theoretical basis and clues for the clinical application of drugs.

\section{Patients and methods}

Methods. In total, 248 cases of silicosis patients with an average age of 50.42 \pm 9.67 years and treated in Branch of Tai'an City Central Hospital (Tai'an, China) from October 2015 to 2017 were retrospectively analyzed in this study, including 226 males and 22 females. Further 116 cases with conventional therapy were the control group, and 132 cases treated with acetylcysteine and tetrandrine tablets on the basis of conventional therapy were the observation group. There was no significant difference in general information between the two groups $(\mathrm{P}>0.05)$. All patients met the criteria for diagnosis and staging of silicosis (13). The cases were excluded due to pregnancy, lactation, other tumors, liver and kidney dysfunction, emphysema, pulmonary tuberculosis and other lung related diseases. Patients with incomplete clinical data or lost to follow-up were also excluded. This study was approved by the Ethics Committee of the hospital. All the subjects were informed and agreed to participate in the clinical study, and the patients and their families signed a fully informed consent form. General information is provided in Table I.

Medicines and instruments. Ambroxol Hydrochloride oral liquid was from Hong Kong Bright Future Pharmaceutical Laboratories Limited (SFDA approval no. H20067008). Xiaokechuan tablet was from the sixth pharmaceutical factory of Harbin Pharmaceutical Group Co., Ltd. (SFDA approval no. Z23021362). Acetylcysteine effervescent tablets and tetrandrine were from Zhejiang Jinhua Conba Biopharma Co., Ltd. (SFDA approval no. H20057334, SFDA approval no. H33022075). British BTL08 lung function instrument was from Chengdu Buywells Science and Technology Co., Ltd.

Treatment methods. All patients stayed at home and stopped working during treatment, avoiding contact with silica dust and severely damaging working environment. All patients took the medicine on time, did not take other health care products or medicines privately, and did not use other means of recovery. The daily intake of the diet was light and varied. The control group was treated with conventional therapy: Dosage of ambroxol hydrochloride oral liquid $10 \mathrm{ml} / \mathrm{time}$, 2 times daily. At the same time, Xiaokechuan tablet were taken orally 4 tablets/time, 3 times daily. The observation group was treated with acetylcysteine and tetrandrine tablets on the basis of routine treatment: Dosage of acetylcysteine effervescent tablets $0.6 \mathrm{~g} / \mathrm{time}, 2$ times daily; dosage of tetrandrine tablets $100 \mathrm{mg} /$ time, 2 times daily. After 6 days, the drug was stopped for 1 day. The two groups were treated with 4 courses for 8 months. The treatment course of the patient was decided by the attending doctor according to the condition of the patient, and the treatment time was adequate (14).

Evaluation of clinical efficacy, adverse reactions and side effects. The respiratory symptoms 1 week before and after treatment of the two groups were evaluated according to the internationally accepted St. George's Respiratory questionnaire (SGRQ) (15), including chest tightness, chest pain, cough and sputum production.

Improvement rate $=$ (number of patients with the above symptoms before treatment, number patients with the above symptoms after treatment)/number of patients with the above symptoms before treatment $\mathrm{x} 100 \%$.

Side effects of the drug include mild lethargy, fatigue, epigastric discomfort, nausea and vomiting.

Evaluation of pulmonary function. Pulmonary function before and after treatment was measured by pulmonary function instrument, including the following indicators: Forced vital capacity (FVC) and forced expiratory volume in $1 \mathrm{sec}$ (FEV1).

Evaluation of exercise tolerance. Exercise tolerance before and after treatment was measured by $6 \mathrm{MWT}$ (16). A straight line of $30 \mathrm{~m}$ long was drawn on the indoor floor with a marker at each end, and the patient controlled the speed and walked along the line to and fro. The walking distance within 6 min was calculated.

Statistical analysis. Statistical analysis was carried out using SPSS 19.0 (IBM, Corp.) software. The count data were expressed as $[\mathrm{n}(\%)]$, and analyzed with the $\chi^{2}$ test. The measurement data were expressed as (mean \pm SD). The data of the two groups were compared by t-test, and the data of multiple groups were compared by Oneway ANOVA. $\mathrm{P}<0.05$, indicates a statistically significant difference.

\section{Results}

Clinical symptoms and adverse reactions of the two groups before and after treatment. The symptoms of chest tightness, chest pain, cough and sputum production in the observation group and the control group after treatment were significantly lower than those before treatment, the difference was statistically significant $(\mathrm{P}<0.05)$. There was no significant difference in chest tightness, chest pain, cough and sputum production between the two groups before treatment $(P>0.05)$. The improvement rate of chest tightness, chest pain, cough and sputum production in the observation group after treatment was significantly higher than that in the control group, and the difference was statistically significant $(\mathrm{P}<0.05)$.

According to the follow-up results, the control group did not have adverse reactions during the treatment period; in the observation group, there were 4 patients with adverse reactions such as nausea, abdominal pain and abdominal distension during treatment. One of them had an increase in transaminase activity, the adverse reaction disappeared 1 month after the 
Table I. General information (n).

\begin{tabular}{|c|c|c|c|c|}
\hline Factors & Observation group $(n=132)$ & Control group $(n=116)$ & $\chi^{2}$ value & P-value \\
\hline Age (years) & & & 0.183 & 0.703 \\
\hline$<50$ & 59 & 55 & & \\
\hline$\geq 50$ & 73 & 61 & & \\
\hline Sex & & & 1.051 & 0.374 \\
\hline Male & 118 & 108 & & \\
\hline Female & 14 & 8 & & \\
\hline Stage & & & 0.267 & 0.878 \\
\hline I & 84 & 76 & & \\
\hline II & 32 & 25 & & \\
\hline III & 16 & 15 & & \\
\hline Dust exposure time (year) & & & 0.283 & 0.678 \\
\hline$<10$ & 94 & 79 & & \\
\hline$\geq 10$ & 38 & 37 & & \\
\hline Complication & & & 1.051 & 0.374 \\
\hline Yes & 14 & 8 & & \\
\hline No & 118 & 108 & & \\
\hline Smoking & & & 0.762 & 0.683 \\
\hline Past smoker & 79 & 65 & & \\
\hline Current smoker & 24 & 20 & & \\
\hline Never smoked & 29 & 31 & & \\
\hline
\end{tabular}

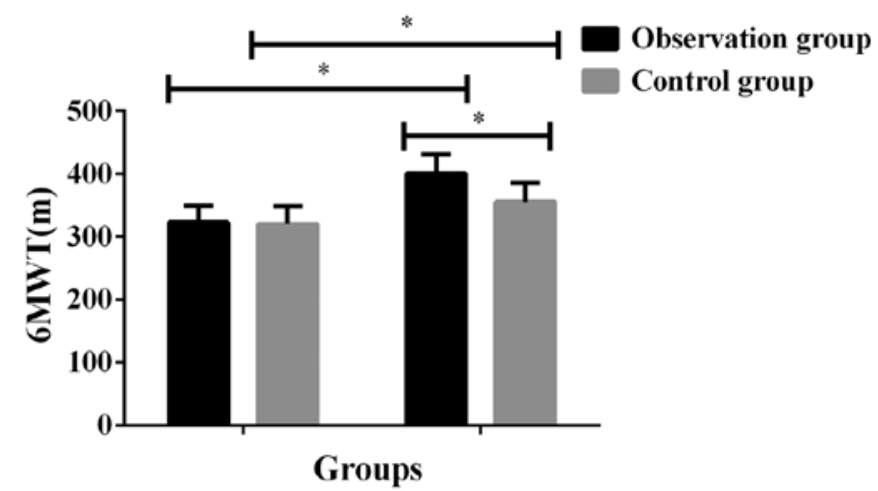

Figure 1. Comparison of exercise tolerance between the two groups before and after treatment. The results showed that the $6 \mathrm{MWT}$ in the observation group and the control group after treatment was significantly higher than that before treatment, and the difference was statistically significant $(\mathrm{P}<0.001)$ There was no significant difference in 6MWT between the two groups before treatment $(\mathrm{P}>0.05)$. The $6 \mathrm{MWT}$ in the observation group after treatment was significantly higher than that in the control group, and the difference was statistically significant $(\mathrm{P}<0.001)$. $\mathrm{P}<0.001$ compared with $6 \mathrm{MWT}$ after treatment in the control group. 6MWT, 6-min walk test.

drug was stopped according to the doctor's advice. According to the results of reexamination, there was no significant changes in ECG, renal function, and blood routine indicators between the observation group and the control group (Table II).

Exercise tolerance and pulmonary function before and after treatment in the groups. The 6MWT, FVC and FEV1 in the observation group and the control group after treatment was significantly higher than that before treatment, and the difference was statistically significant $(\mathrm{P}<0.001)$. There was no significant difference in 6MWT, FVC, FEV1 between the two groups before treatment ( $\mathrm{P}>0.05)$. The 6MWT, FVC and FEV1 in the observation group after treatment was significantly higher than that in the control group, and the difference was statistically significant $(\mathrm{P}<0.001)$ (Table III and Figs. 1 and 2).

Side effects after treatment in the groups. The comparison of the two groups showed that the incidence of drug side effects in the observation group was significantly lower than that in the control group $(\mathrm{P}<0.05)$ (Table IV).

\section{Discussion}

Pulmonary fibrosis is the main clinical manifestation of silicosis which will exacerbate with the prolongation of the course of disease. First of all, the pulmonary blood circulation is blocked, which damages the defense system of the respiratory tract, and results in the decrease of the patient's immune function, the formation of repeated infection, and even irreversible pulmonary failure in severe patients $(17,18)$. Because silicosis has no typical clinical symptoms in the early stage and no special drug treatment, it is already serious when the symptoms of chest tightness, chest pain, cough, sputum production, etc. emerge (19). Airway obstruction of respiratory system in silicosis patients also affects motor function. Therefore, inhibition of pulmonary fibrosis is the main criterion for treatment of silicosis. The efficacy of acetylcysteine combined with tetrandrine tablets on the basis of conventional therapy in the treatment of silicosis was evaluated for clinical efficacy, exercise tolerance and pulmonary function in patients. 
Table II. Clinical symptoms of the both groups before and after treatment (n).

\begin{tabular}{|c|c|c|c|c|}
\hline Indicator & Observation group & Control group & $\chi^{2}$ value & P-value \\
\hline Chest tightness & 132 & 116 & 4.616 & 0.035 \\
\hline Before treatment & 124 & 109 & & \\
\hline After treatment & 77 & 82 & & \\
\hline Improvement rate $(\%)$ & 37.90 & 24.77 & & \\
\hline Chest pain & & & 6.853 & 0.013 \\
\hline Before treatment & 117 & 104 & & \\
\hline After treatment & 62 & 73 & & \\
\hline Improvement rate $(\%)$ & 47.01 & 29.81 & & \\
\hline Cough & & & 17.768 & $<0.001$ \\
\hline Before treatment & 121 & 102 & & \\
\hline After treatment & 35 & 58 & & \\
\hline Improvement rate $(\%)$ & 71.07 & 43.14 & & \\
\hline Sputum production & & & 13.873 & $<0.001$ \\
\hline Before treatment & 128 & 105 & & \\
\hline After treatment & 42 & 60 & & \\
\hline Improvement rate $(\%)$ & 67.19 & 42.86 & & \\
\hline
\end{tabular}
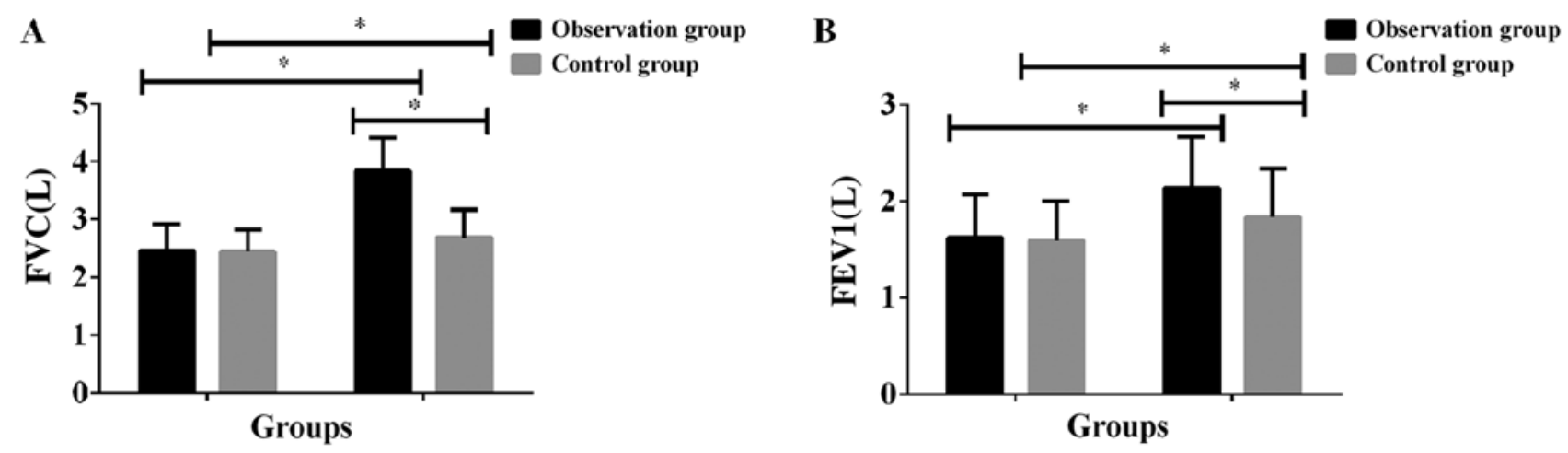

Figure 2. Comparison of pulmonary function between the two groups before and after treatment. The results showed that (A) the FVC in the observation group and the control group after treatment were significantly higher than that before treatment, and the difference was statistically significant $(\mathrm{P}<0.001)$. There was no significant difference in FVC between the two groups before treatment $(\mathrm{P}>0.05)$. FVC in the observation group after treatment was significantly higher than that in the control group, and the difference was statistically significant $(\mathrm{P}<0.001)$. (B) The FEV1 in the observation group and the control group after treatment were significantly higher than those before treatment, and the difference was statistically significant $(\mathrm{P}<0.001)$. There was no significant difference in FVC between the two groups before treatment $(\mathrm{P}>0.05)$. The FEV1 in the observation group after treatment was significantly higher than that in the control group, and the difference was statistically significant $(\mathrm{P}<0.001) .{ }^{*} \mathrm{P}<0.001$ with the FVC and FEV1 in the control group after treatment. $6 \mathrm{MWT}$, 6-min walk test; FVC, forced vital capacity; FEV1, forced expiratory volume in $1 \mathrm{sec}$.

No other studies have been found to verify the effect of acetylcysteine combined with tetrandrine tablets on 6MWT in patients with silicosis. However, this study showed that the $6 \mathrm{MWT}$ in the observation group and the control group after treatment was significantly higher than that before treatment, and the difference was statistically significant $(\mathrm{P}<0.001)$. There was no significant difference in 6MWT between the two groups before treatment $(\mathrm{P}>0.05)$. The $6 \mathrm{MWT}$ in the observation group after treatment was significantly higher than that in the control group, and the difference was statistically significant $(\mathrm{P}<0.001)$. The results showed that the improvement of motor function by the combined treatment of these two drugs was better than that by conventional therapy. In addition, the symptoms of chest tightness, chest pain, cough and sputum production in the observation group and the control group after treatment were significantly lower than those before treatment, and the difference was statistically significant $(\mathrm{P}<0.05)$. There was no significant difference in chest tightness, chest pain, cough and sputum production between the two groups before treatment $(\mathrm{P}>0.05)$. The improvement rate of chest tightness, chest pain, cough and sputum production in the observation group after treatment was significantly higher than that in the control group, and the difference was statistically significant $(\mathrm{P}<0.05)$. The FVC and FEV1 in the observation group and the control group after treatment were significantly higher than those before treatment, and the difference was statistically significant $(\mathrm{P}<0.001)$. There was no significant difference in FVC and FEV1 between the two groups before treatment $(\mathrm{P}>0.05)$. FVC and FEV1 in the observation group after treatment were significantly higher than those in the control group, 
Table III. Exercise tolerance and pulmonary function in both groups before and after treatment.

\begin{tabular}{lclccc}
\hline Groups & Cases & Treatment duration & 6MWT $(\mathrm{m})$ & FVC $(\mathrm{L})$ & FEV1 (L) \\
\hline Observation group & \multirow{2}{*}{132} & Before treatment & $323.24 \pm 25.83$ & $2.45 \pm 0.46$ & $1.62 \pm 0.45$ \\
& & After treatment & $400.67 \pm 31.27^{\mathrm{a}}$ & $3.84 \pm 0.57^{\mathrm{a}}$ & $2.14 \pm 0.52^{\mathrm{a}}$ \\
& & t value & 21.93 & 21.80 & 8.688 \\
& & $<-0.001$ & $<0.001$ & $<0.001$ \\
Control group & \multirow{2}{*}{116} & Before treatment & $320.13 \pm 28.46$ & $2.44 \pm 0.39$ & $1.59 \pm 0.41$ \\
& & After treatment & $355.71 \pm 30.28$ & $2.69 \pm 0.48$ & $1.83 \pm 0.51$ \\
& & t value & 9.222 & 4.354 & 3.950 \\
& & P-value & $<0.001$ & $<0.001$ & $<0.001$ \\
\hline
\end{tabular}

${ }^{\text {aP }}<0.001$ compared with the control group after treatment. 6MWT, 6-min walk test; FVC, forced vital capacity; FEV1, forced expiratory volume in $1 \mathrm{sec}$.

Table IV. Pulmonary function in both groups before and after treatment.

\begin{tabular}{lcccc}
\hline Groups & Cases & Treatment duration & FVC (L) & FEV1 (L) \\
\hline Observation group & \multirow{2}{*}{132} & Before treatment & $2.45 \pm 0.46$ & $1.62 \pm 0.45$ \\
& & After treatment & $3.84 \pm 0.57^{\mathrm{a}}$ & $2.14 \pm 0.52^{\mathrm{a}}$ \\
& & t value & 21.80 & 8.688 \\
& & P-value & $<0.001$ & $<0.001$ \\
Control group & \multirow{2}{*}{116} & Before treatment & $2.44 \pm 0.39$ & $1.59 \pm 0.41$ \\
& & After treatment & $2.69 \pm 0.48$ & $1.83 \pm 0.51$ \\
& t value & 4.354 & 3.950 \\
& P-value & $<0.001$ & $<0.001$ \\
\hline
\end{tabular}

${ }^{\text {aP }}<0.001$ compared with the FVC and FEV1 in the control group after treatment. FVC, forced vital capacity; FEV1, forced expiratory volume in $1 \mathrm{sec}$.

and the difference was statistically significant $(\mathrm{P}<0.001)$. The findings of Weiss et al (20) and Luckhardt et al (21) are consistent with ours. In their studies, the improvement rate of cough, sputum, chest tightness, chest pain and dyspnea after treatment with acetylcysteine combined with tetrandrine was significantly higher than that in the control group; the FVC, FEV1 and FEV1/FVC in the treatment of silicosis with acetylcysteine combined with tetrandrine tablets after treatment were significantly improved compared with those before treatment in the observation group, and the degree of improvement in the observation group was higher than that in the control group, and the difference was statistically significant. These results suggested that acetylcysteine combined with tetrandrine tablets was effective in the treatment of silicosis and could improve the clinical symptoms and pulmonary function. According to literature, the destruction of the structure and function of the tracheal mucosa in silicosis patients is caused by the release of toxic substances by neutrophils in the bronchial mucosa, while acetylcysteine can control the inflammatory factors in patients' serum, and exert antioxidant and antiinflammatory effects to slow down the development of the disease (22). Zhang et al (23) showed that acetylcysteine reduced the reactive oxygen species in rats exposed to silica, and also inhibited the mitochondrial apoptotic pathway, thereby reducing the severity of pulmonary fibrosis. Tetrandrine is a calcium antagonist that blocks the calcium channel of fiber cells and inhibits their proliferation (24). Moreover, it can inhibit collagen synthesis in silicosis tissue and act on cell microtubules to make collagen form fibrous structure only outside the cells, and reduce collagen protein in silicosis tissue and loosen or even degrade the tubercle of patients with silicotic nodules. Tetrandrine can inhibit various diseases such as hypertension, silicosis and arthritis, and has a good prospect of protecting liver cells, resisting liver fibrosis, reducing portal hypertension, inducing tumor cell apoptosis and reversing multidrug resistance (25). The role of tetrandrine in various signal transduction pathways such as reactive oxygen species, enhanced autophagy flux, multidrug resistance reversal, caspase pathway, cell cycle arrest, and calcium channel modification has been demonstrated (26). As a result, the patients with silicotic nodules who received tetrandrine were effectively improved, shadow decreased and faded, and symptoms such as chest tightness and cough were alleviated (27).

In conclusion, acetylcysteine combined with tetrandrine tablets on the basis of conventional therapy in treatment of silicosis is more effective than conventional therapy alone, which can also improve exercise tolerance, pulmonary 
function and clinical symptoms such as chest tightness, chest pain, cough, sputum production of silicosis patients, and is helpful for clinical application.

\section{Acknowledgements}

Not applicable.

\section{Funding}

No funding was received.

\section{Availability of data and materials}

The datasets used and/or analyzed during the present study are available from the corresponding author on reasonable request.

\section{Authors' contributions}

XG and JQ led the conception and design of this study. XG, JQ and HL were responsible for the data collection and analysis. JQ, HL and ZX were in charge of interpreting the data and drafting the manuscript. $\mathrm{XG}$ and $\mathrm{ZX}$ made revision from critical perspective for important intellectual content. All authors read and approved the final manuscript.

\section{Ethics approval and consent to participate}

The study was approved by the Ethics Committee of Branch of Tai'an City Central Hospital (Tai'an, China). Signed informed consents were obtained from the patients and/or guardians.

\section{Patient consent for publication}

Not applicable.

\section{Competing interests}

The authors declare that they have no competing interests.

\section{References}

1. Akgün M and Ergan B: Silicosis in Turkey: Is it an endless nightmare or is there still hope? Turk Thorac J 19: 89-93, 2018.

2. Brhel P, Petrovová M and Lorenzová D: Ventilatory function in patients with silicosis or coal workers' pneumoconiosis. Vnitr Lek 61: 1023-1027, 2015 (In Czech).

3. Rimal B, Greenberg AK and Rom WN: Basic pathogenetic mechanisms in silicosis: Current understanding. Curr Opin Pulm Med 11: 169-173, 2005.

4. Richter K and Kietzmann T: Reactive oxygen species and fibrosis: Further evidence of a significant liaison. Cell Tissue Res 365: 591-605, 2016.

5. Peng HB, Wang RX, Deng HJ, Wang YH, Tang JD, Cao FY and Wang JH: Protective effects of oleanolic acid on oxidative stress and the expression of cytokines and collagen by the AKT/NFKB pathway in silicotic rats. Mol Med Rep 15: 3121-3128, 2017.

6. Huaux F: New developments in the understanding of immunology in silicosis. Curr Opin Allergy Clin Immunol 7: 168-173, 2007.

7. Gurjar D, Gupta M, Sharma H and Pathak S: Study to find out the efficacy of 6 minute walk test in assessing response to medical intervention in chronic obstructive pulmonary disease patients IJAM 3: 3, 2019
8. Saglam M, Vardar-Yagli N, Savci S, Inal-Ince D, Calik-Kutukcu E, Arikan H and Coplu L: Functional capacity, physical activity, and quality of life in hypoxemic patients with chronic obstructive pulmonary disease. Int $\mathbf{J}$ Chron Obstruct Pulmon Dis 10: 423-428, 2015.

9. Yeh ST, Guo HR, Su YS, Lin HJ, Hou CC, Chen HM, Chang MC and Wang YJ: Protective effects of nacetylcysteine treatment post acute paraquat intoxication in rats and in human lung epithelial cells. Toxicology 223: 181-190, 2006.

10. McLaughlin MF, Donoviel DB and Jones JA: Novel indications for commonly used medications as radiation protectants in spaceflight. Aerosp Med Hum Perform 88: 665-676, 2017.

11. Li FQ, Lu B, Chen WB and Yang H: Tetrandrine loaded sustainedrelease microcapsules for lung targeting. Yao Xue Xue Bao 36: 220-223, 2001 (In Chinese)

12. Liu C, Gong X, Xiao X, Yuan X and Cai B: Effects of tetrandrine combined with large volume whole lung lavage on the quality of life and oxidative stress of pneumoconiosis patients. Zhonghua Lao Dong Wei Sheng Zhi Ye Bing Za Zhi 32: 219-221, 2014 (In Chinese).

13. Suut S, Al-Ani Z, Allen C, Rajiah P, Durr-E-Sabih, Al-Harbi A, Al-Jahdali $\mathrm{H}$ and Khan AN: Pictorial essay of radiological features of benign intrathoracic masses. Ann Thorac Med 10: 231-242, 2015.

14. Reychler G, Audag N, Mestre NM and Caty C: Assessment of validity and reliability of the 1-minute sit-to-stand test to measure the heart rate response to exercise in healthy children. JAMA pediatr 173: 692-693, 2019.

15. Ringbaek T, Martinez G and Lange P: A comparison of the assessment of quality of life with CAT, CCQ, and SGRQ in COPD patients participating in pulmonary rehabilitation. COPD 9: 12-15, 2012.

16. Ora J, Calzetta L, Pezzuto G, Senis L, Paone G, Mari A, Portalone S, Rogliani P, Puxeddu E and Saltini C: A 6MWT index to predict $\mathrm{O}_{2}$ flow correcting exercise induced $\mathrm{SpO}_{2}$ desaturation in ILD. Respir Med 107: 20142021, 2013.

17. Huff JE, Dinger CY, Kline BW, Whitfield BL and Hammons AS: A health view of asbestos: An annotated literature collection. 1960-1974. Environ Health Perspect 9: 341-462, 1975.

18. Zhang Y, Yang F, Liu Y, Peng HB, Geng YC, Li SF, Xu H, Zhu LY, Yang XH and Brann D: Influence of the interaction between Ac-SDKP and Ang II on the pathogenesis and development of silicotic fibrosis. Mol Med Rep 17: 7467-7476, 2018.

19. Wang SX, Liu P, Wei MT, Chen L, Guo Y, Wang RY, Tu ZG and Liang XC: Roles of serum clara cell protein 16 and surfactant protein $\mathrm{D}$ in the early diagnosis and progression of silicosis. J Occup Environ Med 49: 834-839, 2007.

20. Weiss DJ, Chambers D, Giangreco A, Keating A, Kotton D, Lelkes PI, Wagner DE and Prockop DJ; ATS Subcommittee on Stem Cells and Cell Therapies: An official American Thoracic Society workshop report: Stem cells and cell therapies in lung biology and diseases. Ann Am Thorac Soc 12: 79-97, 2015.

21. Luckhardt TR, Müller-Quernheim J and Thannickal VJ: Update in diffuse parenchymal lung disease 2011. Am J Respir Crit Care Med 186: 24-29, 2012.

22. Ning JX, Zhang LP and Cui Y: Evaluation of clinical efficacy of tretrandrine combined with acetylcysteine effervescent tablets in the treatment of silicosis. Zhonghua Lao Dong Wei Sheng Zhi Ye Bing Za Zhi 35: 298-299, 2017 (In Chinese).

23. Zhang L, He YL, Li QZ, Hao XH, Zhang ZF, Yuan JX, Bai YP, Jin YL, Liu N, Chen G, et al: Nacetylcysteine alleviated silica induced lung fibrosis in rats by downregulation of ROS and mitochondrial apoptosis signaling. Toxicol Mech Methods 24: 212-219, 2014.

24. Li C, Lu Y, Du S, Li S, Zhang Y, Liu F, Chen Y, Weng D and Chen J: Dioscin exerts protective effects against crystalline silica-induced pulmonary fibrosis in mice. Theranostics 7: 4255-4275, 2017.

25. Cai XH, Wang S and Chen BA: Research advances on the pharmacological effects of tetrandrine. Chin J Nat Med 9: 473-480, 2011.

26. Bhagya $\mathrm{N}$ and Chandrashekar KR: Tetrandrine - A molecule of wide bioactivity. Phytochemistry 125: 5-13, 2016.

27. Weiss DJ, Bates JH, Gilbert T, Liles WC, Lutzko C, Rajagopal J and Prockop D: Stem cells and cell therapies in lung biology and diseases: Conference report. Ann Am Thorac Soc 10: S25-S44, 2013. 